\title{
Use of a Gonadotropin-Releasing Hormone Analog for Protection Against Premature Ovarian Failure During Cyclophosphamide Therapy in Women With Severe Lupus
}

\author{
Emily C. Somers, ${ }^{1}$ Wendy Marder, ${ }^{2}$ Gregory M. Christman, ${ }^{2}$ Vladimir Ognenovski, ${ }^{2}$ \\ and W. Joseph McCune ${ }^{2}$
}

Objective. Cyclophosphamide (CYC) therapy for systemic lupus erythematosus (SLE), a disease predominantly affecting women of childbearing age, causes an unacceptably high incidence of irreversible premature ovarian failure (POF). This study was performed to evaluate the effectiveness of depot leuprolide acetate, a synthetic gonadotropin-releasing hormone analog (GnRH-a), for protection against POF during CYC therapy.

Methods. Young women with severe SLE treated in a standardized protocol of monthly intravenous bolus CYC were offered treatment with GnRH-a (depot leuprolide acetate; a 3.75-mg monthly injection during the standard CYC regimen). Patients treated with GnRH-a were compared with controls individually matched by age $( \pm 5$ years) and by cumulative CYC dose $( \pm 5 \mathrm{gm})$. Reproductive status was determined after a minimum followup of 3 years after CYC therapy. The primary outcome was time to POF. Paired summary statistical analyses, Kaplan-Meier survival estimates, and Cox regression analyses were performed to assess differences in outcome between groups.

Results. POF developed in 1 of 20 women treated with GnRH-a (5\%) compared with 6 of 20 controls (30\%)

Supported by the Herb and Carol Amster Lupus Research Fund, the University of Michigan Multipurpose Arthritis Center (grant 5-P60-AR-20557), the University of Michigan General Clinical Research Center (grant M01-RR-00042), and the University of Michigan Rheumatic Disease Core Center (grant 1-P30-48310).

${ }^{1}$ Emily C. Somers, ScM: University of Michigan Health System, Ann Arbor, and London School of Hygiene and Tropical Medicine, London, UK; ${ }^{2}$ Wendy Marder, MD, Gregory M. Christman, MD, Vladimir Ognenovski, MD, W. Joseph McCune, MD: University of Michigan Health System, Ann Arbor.

Address correspondence and reprint requests to W. Joseph McCune, MD, 3918 Taubman Center, 1500 East Medical Center Drive, Ann Arbor, MI 48109-0358. E-mail: jmccune@umich.edu.

Submitted for publication January 24, 2005; accepted in revised form June 3, 2005. matched by age and cumulative CYC dose (matched odds ratio $0.09, P<0.05)$. Kaplan-Meier estimates demonstrated improved cumulative ovarian protection over time in the GnRH-a-treated group $(P=0.04)$.

Conclusion. Treatment with GnRH-a during CYC therapy was associated with a significant reduction of POF in young women with severe SLE.

Cyclophosphamide (CYC) administration prolongs survival and reduces end-organ damage in patients with severe rheumatic diseases such as systemic lupus erythematosus (SLE), Wegener's granulomatosis, and polyarteritis nodosa. However, treatment with CYC and other alkylating agents such as chlorambucil is associated with an unacceptably high incidence of significant toxicities, including premature ovarian failure (POF; hypergonadotropic hypogonadism), with consequent irreversible amenorrhea and infertility (1-3). This has particular significance in the SLE population, since lupus disproportionately affects women of reproductive age. In experimental animals, CYC exposure results in DNA crosslinking in granulosa cells, reduced numbers of granulosa cells, decreased circulating levels of progesterone and estrogen, and ovarian fibrosis $(4,5)$. In humans, CYC-induced damage to the ovary is generally regarded as cumulative and irreversible, due in part to progressive reduction of a limited number of cells (2).

The incidence of POF in both the rheumatic disease and cancer populations treated with $\mathrm{CYC}$ ranges from $12 \%$ to $83 \%$ depending on variables such as patient characteristics and mode of administration. Both age at initiation of therapy and the cumulative CYC dose are strong predictors of POF (1-3,6-9). A study of breast cancer patients showed that the cumulative CYC doses associated with a $50 \%$ incidence of ovarian failure were $20.4 \mathrm{gm}, 9.3 \mathrm{gm}$, and $5.2 \mathrm{gm}$ among women in their 20s, 30 s, and 40s, respectively (10). 
In women of reproductive age, pulsatile secretion of gonadotropin-releasing hormone $(\mathrm{GnRH})$ by the hypothalamus is required to stimulate pituitary gland secretion of follicle-stimulating hormone (FSH) and luteinizing hormone to maintain normal menstrual cycles. Continuous, rather than pulsatile, exposure of the pituitary gland to GnRH suppresses ovulation, reducing estrogen and progesterone to prepubertal levels. This state of reversible "medical castration" can be achieved with externally administered GnRH analogs (GnRH-a). GnRH-a administration during chemotherapy may confer ovarian protection due to ovarian suppression or decline in ovarian blood flow, resulting in decreased exposure of the ovaries to chemotherapeutic agents (11). In animals, continuous administration of GnRH-a decreases incorporation of tritiated thymidine into granulosa cells and prevents CYC-induced ovarian damage $(4,12,13)$. GnRH analogs may also exert direct protective effects on the ovary via peripheral GnRH receptors, which have been documented on human granulosa cells found in developing and mature follicles (14).

Pilot studies using GnRH-a for ovarian protection have demonstrated encouraging results $(2,15-17)$. We report the first study of GnRH-a for ovarian protection in young women with severe lupus undergoing a standardized CYC protocol.

\section{PATIENTS AND METHODS}

This research was approved by the University of Michigan Institutional Review Board. Written informed consent was obtained from research participants.

Intravenous CYC (IVCYC) treatment protocol. The GnRH-a study was nested in a larger IVCYC protocol that has been ongoing since 1985. All study participants underwent a standardized IVCYC protocol for the treatment of severe manifestations of SLE (e.g., nephritis, cerebritis, or vasculitis judged to require addition of immunosuppressive therapy to a steroid regimen to prevent end-organ damage). This sequential therapy protocol consisted of monthly IV boluses of CYC plus a tapering dosage of daily oral prednisone, as previously described (18). Following 6 monthly treatments, patients who achieved satisfactory disease control were switched from IVCYC to an additional 2 years of treatment with either azathioprine or mycophenolate mofetil to reduce toxicity. Those patients who did not achieve satisfactory disease control were treated with an additional 4 monthly boluses of CYC and reevaluated as above. Depot medroxyprogesterone acetate (Upjohn, Kalamazoo, MI) $150 \mathrm{mg}$ parenterally every 3 months was prescribed for contraception during CYC therapy.

GnRH-a protocol for ovarian protection. Participation in the GnRH-a protocol was offered to consecutive female SLE patients in whom IVCYC treatment was initiated from 1993 onward. Depot leuprolide acetate (TAP Pharmaceuticals, Lake Forest, IL), a GnRH agonist, was administered by injection once per month at a dose of $3.75 \mathrm{mg}$ throughout the course of CYC treatment. In patients who did not achieve satisfactory disease control, GnRH-a administration was continued throughout CYC therapy. In order to avoid CYC exposure during the initial surge of estrogen, the GnRH-a injection was timed to occur at least 10 days prior to the subsequent monthly bolus of IVCYC. In the majority of patients, initiation of CYC treatment was indicated on an urgent basis, which did not allow an adequate opportunity to initiate GnRH-a treatment until 10 days prior to the second CYC bolus. Following 4 weeks of therapy, patients without contraindication were given an estradiol patch (estradiol transdermal system, 0.05 or $0.1 \mathrm{mg}$; Novartis Pharmaceuticals, East Hanover, NJ) to maintain estrogen at slightly less than early follicular phase levels and to reduce symptoms of hormonal withdrawal. The transdermal method was chosen over oral estradiol add-back therapy because it avoids increased production of clotting factors resulting from first-pass hepatic metabolism (19).

Study population for analysis of GnRH-a for protection against POF. Patients. Patients were eligible for this study if they had a diagnosis consistent with lupus or if they satisfied the American College of Rheumatology (ACR) criteria for SLE $(20,21)$, were women of reproductive age, and had an exacerbation of disease activity requiring treatment with at least 6 monthly boluses of IVCYC. Patients were excluded from this analysis if they were age $\geq 35$ years at the beginning of CYC treatment or if they were found at baseline to have symptoms consistent with ovarian failure based on gynecologic evaluation.

Controls. Controls were randomly selected female SLE patients in the Michigan Lupus Cohort who had participated in the IVCYC protocol and fulfilled the above eligibility criteria, but who had not received GnRH-a. Specifically, the list of eligible female patients who had received IVCYC but not GnRH-a was sorted by cumulative CYC dose and patient age at the beginning of the induction period. For each patient treated with leuprolide acetate, we matched 1 control patient within \pm 5 years of age and within \pm 5 cumulative gm of CYC, using a stratified random sampling procedure without replacement. The matching was performed in a blinded manner by an investigator (ECS) who was unaware of the reproductive outcomes of the patients.

Assessment of ovarian function. Annual gynecologic evaluation is the standard of care implemented at our institution for SLE patients receiving cytotoxic therapy, particularly given the increased risk for gynecologic malignancies reported in this population $(22,23)$. For the purposes of this study, the preservation of ovarian function was established by the presence of normal menses and/or conception after treatment with the IVCYC protocol. Levels of FSH were routinely assessed as an objective measure of POF in women with oligomenorrhea or amenorrhea, or when early menopause was otherwise suspected based on symptoms or gynecologic examination. Women using cyclic oral contraceptives (OCs) on an ongoing basis were queried for the presence of symptoms consistent with menopause (e.g., hot flashes, vaginal dryness, insomnia) during the pill-free interval. Findings consistent with POF were subsequently confirmed by documentation of amenorrhea of at least 12 months' duration and an FSH level $\geq 40 \mathrm{mIU} / \mathrm{ml}$. The time for the "event" of POF was considered the onset of 
amenorrhea, confirmed after 12 consecutive months. This approach is consistent with that used for calculation of menopausal age in epidemiologic studies (24). Patient followup was not continued past age 40 years, which is considered a standard cutoff age for premature menopause (25). Therefore, cases of ovarian failure in this study could not be attributed to natural menopause.

Statistical analysis. Baseline characteristics were summarized for each treatment group and compared using paired 2 -tailed $t$-tests for continuous variables when the underlying distributions were normal. Fisher's exact test was used for the comparison of categorical variables. Odds ratios (ORs) for matched data were computed based on discordant pairs using a correction factor of 0.5 per cell to account for a cell with zero frequency; McNemar's test for paired data was used to test for significance. To account for followup time, Kaplan-Meier survival estimates, stratified by treatment group, were performed to model the cumulative preservation of ovarian function. Followup time was calculated as the time from $\mathrm{CYC}$ initiation until the first occurrence of one of the following: development of ovarian failure, loss to followup, death, or censoring either at age 40 years or at the end of the followup period. The equality of the survival function across groups was tested for significance using a Cox regression-based variation of the log rank test which accounted for the paired nature of the data. Cox proportional hazards regression was performed to estimate the hazard ratio of ovarian failure. Data management and analysis were performed using Stata 7 (Stata, College Station, TX).

\section{RESULTS}

A total of 40 women (20 treated with GnRH-a and 20 matched controls) were included in the analysis. Thirty-nine patients fulfilled the ACR criteria for SLE; 1 patient initially presented with mononeuritis multiplex and other features characteristic of SLE, but was subsequently diagnosed as having systemic vasculitis. The minimum period of followup was 3.0 years unless ovarian failure developed sooner. The analysis was based on a total of 287.1 person-years at risk for POF, including 186.9 person-years among controls (median 10.3 years at risk for POF, range 0.8-16.7 years) and 100.2 personyears among $\mathrm{GnRH}$-a-treated patients (median 4.6 years at risk for POF, range 0.6-9.3 years). As shown in Table 1, baseline characteristics were similar between the 2 groups, particularly for the matching variables of age at CYC initiation and cumulative CYC dose. The population was relatively young, with ages ranging from 17 years to 32 years at the start of CYC therapy.

At followup, ovarian failure had developed in 1 of $20 \mathrm{GnRH}-\mathrm{a}$-treated patients (5\%) compared with 6 of 20 controls $(30 \%)$. Based on a matched pairs analysis, the odds of ovarian failure were significantly lower in the GnRH-a-treated group (OR 0.09, $P<0.05$ ). The single
Table 1. Baseline characteristics of the study population, stratified by GnRH-a treatment*

\begin{tabular}{lcr}
\hline & $\begin{array}{c}\text { GnRH-a-treated } \\
\text { patients } \\
(\mathrm{n}=20)\end{array}$ & $\begin{array}{r}\text { Control } \\
\text { patients } \\
(\mathrm{n}=20)\end{array}$ \\
\hline $\begin{array}{l}\text { Age at initiation of CYC, mean } \\
\quad \text { SD years }\end{array}$ & $23.9 \pm 1.0$ & $25.0 \pm 0.9$ \\
Cumulative dose of CYC, gm & $12.9 \pm 1.5$ & $12.9 \pm 1.5$ \\
SLE duration, mean \pm SD years & $3.6 \pm 1.2$ & $5.1 \pm 1.5$ \\
Indication for CYC & $16(80)$ & $16(80)$ \\
$\quad$ Renal & $2(10)$ & $3(15)$ \\
CNS & $2(10)$ & $1(5)$ \\
Other & $4(20)$ & $5(25)$ \\
Race & $14(70)$ & $14(70)$ \\
$\quad$ African American & $2(10)$ & $1(5)$ \\
$\quad$ White & & \\
Other &
\end{tabular}

${ }^{*}$ Except where indicated otherwise, values are the number $(\%)$ of patients. There were no significant differences between the groups. $\mathrm{GnRH}-\mathrm{a}=$ gonadotropin-releasing hormone analog; $\mathrm{CYC}=$ cyclophosphamide; SLE = systemic lupus erythematosus; CNS = central nervous system.

GnRH-a-treated patient who developed ovarian failure was older (28.2 years) and received a higher cumulative CYC dose $(33.5 \mathrm{gm})$ than the corresponding mean values for the population (24.4 years and $12.9 \mathrm{gm}$ ), representing the 75th and 99th percentiles of age and dose, respectively. This patient's matched control was concordant for development of ovarian failure. Accounting for time at risk for ovarian failure, Kaplan-Meier survival estimates (Figure 1) showed greater cumulative preservation of ovarian function in the $\mathrm{GnRH}$-a-treated

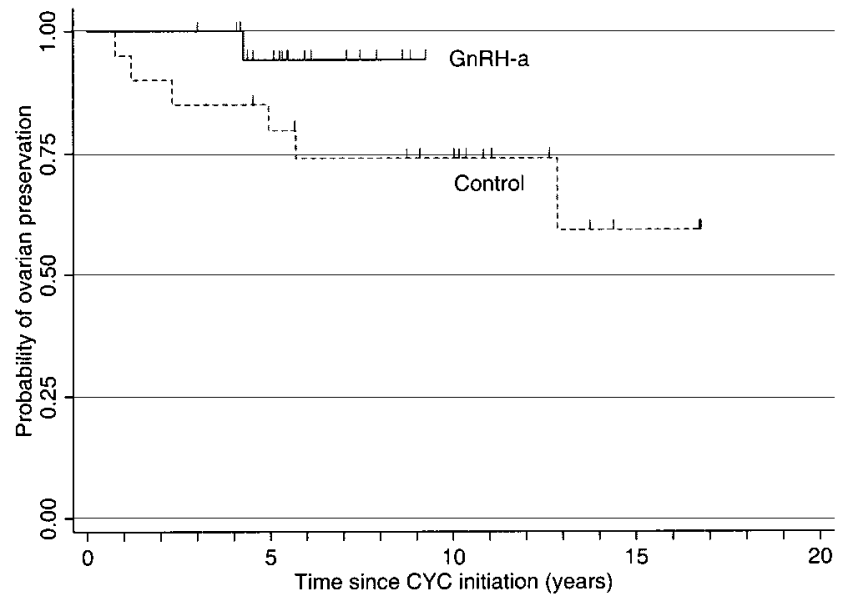

Figure 1. Kaplan-Meier survival estimates of time to premature ovarian failure (POF). Tick marks indicate censored observations (i.e., the final point of followup for patients who did not develop the end point of POF). GnRH-a = gonadotropin-releasing hormone analog; $\mathrm{CYC}=$ cyclophosphamide. 
Table 2. Adverse events potentially associated with the GnRH-a protocol* $^{*}$

\begin{tabular}{lcc}
\hline & $\begin{array}{c}\text { GnRH-a-treated } \\
\text { patients } \\
(\mathrm{n}=20)\end{array}$ & $\begin{array}{c}\text { Control } \\
\text { patients } \\
(\mathrm{n}=20)\end{array}$ \\
\hline Dysfunctional uterine bleeding & $5(25) \dagger$ & $2(10)$ \\
Deep venous thrombosis & $0(0)$ & $1(5)$ \\
Depression & $3(15)$ & $2(10)$ \\
Stroke & $0(0)$ & $0(0)$ \\
\hline
\end{tabular}

* Values are the number $(\%)$ of patients. There were no significant differences between the groups. GnRHa = gonadotropin-releasing hormone analog.

$\dagger$ Includes 2 cases of severe vaginal bleeding associated with thrombocytopenia.

group than in controls $(P=0.04)$. The median time to onset of ovarian failure was 4.3 years (interquartile range 1.2-5.7). Based on Cox regression, the hazard of developing ovarian failure within 10 years of CYC initiation in the GnRH-a-treated group was less than one-tenth that in the control group (hazard ratio 0.09, $95 \%$ confidence internal $0.01-0.8$ ). Although it is not known how many of the women attempted conception subsequent to CYC therapy, 3 of 20 control patients $(15 \%)$ and 7 of $20 \mathrm{GnRH}-a-$ treated patients $(35 \%)$ had successful pregnancies following treatment.

There was no statistically significant difference in adverse events potentially attributable to the study protocol, including dysfunctional uterine bleeding, deep venous thrombosis, or new ischemic cardiac events during the treatment period (Table 2). However, 2 patients in the GnRH-a-treated group experienced severe dysfunctional uterine bleeding and severe thrombocytopenia. In the GnRH-a-treated group, adverse events not attributable to the study protocol included a retroperitoneal bleed in 1 patient, an episode of hematuria in 1 patient, and development of condylomata and a dystonic reaction of unknown cause in 1 patient. In the control group, 1 patient developed superficial thrombophlebitis and 1 patient developed osteonecrosis.

At long-term followup, there were 5 deaths ( 4 of 20 patients in the control group and 1 of 20 patients in the GnRH-a-treated group). All deaths occurred a minimum of 5.5 years after IVCYC initiation. The causes of death were myocardial infarction $(\mathrm{n}=1)$, sudden cardiac death $(\mathrm{n}=3)$, and sepsis $(\mathrm{n}=1)$.

\section{DISCUSSION}

In this study of young women treated with IVCYC for severe manifestations of lupus, administra- tion of a depot GnRH-a was associated with a significant reduction of POF. Only 1 of 20 patients in the GnRHa-treated group (5\%) developed ovarian failure compared with 6 of 20 controls (30\%). Notably, the 1 patient who developed POF in the GnRH-a-treated group was in the 75th percentile of age and in the 99th percentile of cumulative CYC dose for our study population, and thus this patient was at particularly high risk for POF. This is the first study to analyze a large group of lupus patients with controls individually matched for age and cumulative dose of CYC, all of whom were undergoing comparable treatment protocols except for $\mathrm{GnRH}-\mathrm{a}$ use. Our study was also unique in that it included add-back estradiol therapy, demonstrating that the protective effect of GnRH-a is not merely the result of a hypoestrogenic environment.

Previous studies have demonstrated promising results for GnRH-a cotherapy, although all have had important limitations, including heterogeneous chemotherapy regimens with varying degrees of cytotoxicity, limited comparability of control groups with GnRH-a recipients, and failure to incorporate time at risk for ovarian failure into their analyses. In 3 studies by Blumenfeld et al $(2,15,16)$, reduced ovarian injury was reported in women receiving GnRH-a during chemotherapy. The study populations included at least 8 SLE patients as well as lymphoma and leukemia patients. A study of Hodgkin's disease patients receiving standard MVPP (nitrogen mustard, vinblastine, procarbazine, prednisone) chemotherapy did not reveal evidence for ovarian protection among 8 women receiving GnRH-a cotreatment (intranasal buserelin) compared with 10 controls (26). However, a study of teenage and young adult patients with lymphoma undergoing various polychemotherapy regimens showed that $100 \%$ of 12 patients receiving GnRH-a cotreatment resumed menstruation compared with none of 4 controls (17).

Our treatment protocol, which uses monthly bolus CYC to achieve remission of lupus nephritis followed by maintenance therapy with azathioprine or mycophenolate mofetil, is consistent with current sequential therapy regimens designed to minimize cumulative CYC exposure in lupus patients (27). Despite the shift in current treatment patterns toward shorter CYC courses, a relatively high incidence of ovarian failure persists (28). Almost one-third of our control patients developed POF, despite a mean cumulative CYC exposure of 12.9 gm. This contrasts with cumulative doses that have been routinely administered for rheumatic diseases in the past ( $\sim 30 \mathrm{gm}$ for a 3-year course of IVCYC for lupus 
nephritis [28], and up to $100 \mathrm{gm}$ oral CYC for Wegener's granulomatosis [29]).

Our regimen for ovarian protection was well tolerated. Patients who developed classic symptoms of hypoestrogenism with GnRH-a therapy, including vaginal dryness and hot flashes $(30,31)$, appeared to respond to reinstitution of concomitant estrogen replacement therapy. Women who receive GnRH-a usually develop amenorrhea with decreased hormone levels within 3-8 weeks (32), with return of menses $\sim 6$ weeks after the last subcutaneous injection or 10 weeks after a monthly depot injection. During the weeks after initiation of therapy, we found no evidence of SLE flares potentially attributable to the temporary rise in estrogen.

Of greater concern with GnRH-a therapy is the accelerated loss of estrogen-dependent trabecular bone, which has been established in studies of GnRH-a for treatment of endometriosis (33). The extent to which restoration of normal bone density occurs after cessation of therapy is controversial. Two studies of the reversibility of bone loss resulting from GnRH-a therapy have shown complete reversal after 4 months (34) and incomplete recovery after 12 months (35). These studies included women with normal baseline bone densities, and they may not be applicable to patients with lupus or other connective tissue diseases with a high prevalence of osteoporosis $(36,37)$.

Measurements of bone mineral density before and after treatment were not included in this study. However, to minimize loss of bone mineral density in our treatment group, and to prevent symptoms of estrogen withdrawal, we used a patch to replace estradiol at or below expected physiologic levels when not otherwise contraindicated. Quarterly injections of depot medroxyprogesterone provided reliable contraception and prevented action of unopposed estrogen on the uterus. Use of antiresorptive agents to protect against bone loss may be important to consider in patients receiving both corticosteroids and medications such as leuprolide acetate or depot medroxyprogesterone that decrease estrogen levels, particularly if add-back estrogen therapy is contraindicated.

Despite potential drawbacks associated with GnRH-a, this strategy is less invasive and costly than surgical procedures under investigation for fertility preservation during immunosuppressive therapy, such as cryopreservation of unfertilized ova or ovarian tissue. Although promising, oocyte cryopreservation prior to chemotherapy is considered experimental by the American Society for Reproductive Medicine (38) and should be performed under the auspices of an institutional review board. Furthermore, procedures involving ovarian stimulation and oocyte retrieval may be associated with high risks among SLE patients, in whom blood dyscrasias and use of immunosuppressive therapy are common. Cryopreservation of embryos, although well established and readily available, similarly suffers from great expense and the added risks of supraphysiologic estradiol levels with stimulation during an acute flare of SLE. Moreover, applicability of this approach is limited by the requirement for patients to have a partner available and willing to create embryos.

OC pills have also been considered for ovarian protection during chemotherapy, but supportive data are lacking. In a widely cited, uncontrolled study of a series of 6 patients receiving OC pills during standard MVPP treatment for Hodgkin's disease, menses resumed in 5 patients; the sixth patient continued taking OC pills after chemotherapy (39). However, a more recent study comparing 31 OC pill-treated patients with 90 matched non-OC pill-treated patients receiving chemotherapy showed no difference in the rate of ovarian failure, using rates of early menopause and pregnancies as outcome measures (40).

While our study is limited because it was not a randomized controlled trial, we matched controls to account for known confounders. It is possible that there are unknown factors that we could not adjust for, but the baseline characteristics of the 2 groups appear balanced. Another concern is that the controls tended to have begun CYC therapy prior to the availability of the GnRH-a protocol, and thus they accrued longer followup as a group. This was mitigated by using time-toevent analysis and by including only patients with at least 3 years of followup. Although POF often develops within this time frame, it will nonetheless be important to perform further long-term followup on the study population.

Control patients who received IVCYC at an earlier time than GnRH-a-treated patients could have been more severely ill because of the evolution of clinical practice in our institution toward using this treatment earlier for renal disease. However, there are no data to suggest that the severity of lupus activity affects the development of CYC-induced damage to the ovary. An additional limitation is that our study was not designed to assess preservation of ovarian reserve. Because depot medroxyprogesterone acetate was used in our protocol, the possibility cannot be excluded that this agent in combination with GnRH-a contributed to protection against POF. However, there is no evidence to suggest that depot medroxyprogesterone acetate would 
be effective in this regard. Lack of an independent effect of depot medroxyprogesterone for protection against POF is further substantiated by the high rate of POF in our controls, despite their use of depot medroxyprogesterone for contraception.

In this study, treatment with a depot GnRH-a during CYC therapy was associated with a significant reduction in the future incidence of ovarian failure among women with severe SLE. Sequential therapy consisting of CYC induction followed by maintenance treatment is becoming the standard of care for severe lupus nephritis, lending particular relevance to our findings of ovarian protection in the context of a modern immunosuppressive protocol with lengthy followup. Further investigations are needed to confirm the efficacy and mechanism of action of GnRH-a for ovarian protection during CYC therapy. Given the lack of proven alternatives for preventing CYC-induced ovarian injury, this intervention has strong potential as a safe, costeffective, and easily administered method for ovarian preservation in women undergoing chemotherapy for a wide variety of indications.

\section{REFERENCES}

1. Boumpas DT, Austin HA III, Vaughan EM, Yarboro CH, Klippel $\mathrm{JH}$, Balow JE. Risk for sustained amenorrhea in patients with systemic lupus erythematosus receiving intermittent pulse cyclophosphamide therapy. Ann Intern Med 1993;119:366-9.

2. Blumenfeld Z, Shapiro D, Shteinberg M, Avivi I, Nahir M. Preservation of fertility and ovarian function and minimizing gonadotoxicity in young women with systemic lupus erythematosus treated by chemotherapy. Lupus 2000;9:401-5.

3. Warne GL, Fairley KF, Hobbs JB, Martin FI. Cyclophosphamideinduced ovarian failure. N Engl J Med 1973;289:1159-62.

4. Ataya KM, Valeriote FA, Ramahi-Ataya AJ. Effect of cyclophosphamide on the immature rat ovary. Cancer Res 1989;49: $1660-4$.

5. Slater CA, Liang MH, McCune WJ, Christman GM, Laufer MR. Preserving ovarian function in patients receiving cyclophosphamide. Lupus 1999;8:3-10.

6. Mok CC, Lau CS, Wong RW. Risk factors for ovarian failure in patients with systemic lupus erythematosus receiving cyclophosphamide therapy. Arthritis Rheum 1998;41:831-7.

7. Ioannidis JP, Katsifis GE, Tzioufas AG, Moutsopoulos HM. Predictors of sustained amenorrhea from pulsed intravenous cyclophosphamide in premenopausal women with systemic lupus erythematosus. J Rheumatol 2002;29:2129-35.

8. McDermott EM, Powell RJ. Incidence of ovarian failure in systemic lupus erythematosus after treatment with pulse cyclophosphamide. Ann Rheum Dis 1996;55:224-9.

9. Langevitz P, Klein L, Pras M, Many A. The effect of cyclophosphamide pulses on fertility in patients with lupus nephritis. Am J Reprod Immunol 1992;28:157-8.

10. Koyama H, Wada T, Nishizawa Y, Iwanaga T, Aoki Y. Cyclophosphamide-induced ovarian failure and its therapeutic significance in patients with breast cancer. Cancer 1977;39:1403-9.

11. Blumenfeld Z. Gynaecologic concerns for young women exposed to gonadotoxic chemotherapy. Curr Opin Obstet Gynecol 2003; 15:359-70.

12. Karashima T, Zalatnai A, Schally AV. Protective effects of analogs of luteinizing hormone-releasing hormone against chemotherapyinduced testicular damage in rats. Proc Natl Acad Sci U S A 1988;85:2329-33.

13. Ataya KM, McKanna JA, Weintraub AM, Clark MR, LeMaire WJ. A luteinizing hormone-releasing hormone agonist for the prevention of chemotherapy-induced ovarian follicular loss in rats. Cancer Res 1985;45:3651-6.

14. Peng C, Fan NC, Ligier M, Vaananen J, Leung PC. Expression and regulation of gonadotropin-releasing hormone $(\mathrm{GnRH})$ and GnRH receptor messenger ribonucleic acids in human granulosaluteal cells. Endocrinology 1994;135:1740-6.

15. Blumenfeld Z, Avivi I, Linn S, Epelbaum R, Ben-Shahar M, Haim N. Prevention of irreversible chemotherapy-induced ovarian damage in young women with lymphoma by a gonadotrophin-releasing hormone agonist in parallel to chemotherapy. Hum Reprod 1996;11:1620-6.

16. Blumenfeld Z. Ovarian rescue/protection from chemotherapeutic agents. J Soc Gynecol Investig 2001;8:S60-4.

17. Pereyra Pacheco B, Mendez Ribas JM, Milone G, Fernandez I, Kvicala R, Mila T, et al. Use of GnRH analogs for functional protection of the ovary and preservation of fertility during cancer treatment in adolescents: a preliminary report. Gynecol Oncol 2001;81:391-7.

18. McCune WJ, Golbus J, Zeldes W, Bohlke P, Dunne R, Fox D. Clinical and immunologic effects of monthly intravenous cyclophosphamide in severe systemic lupus. N Engl J Med 1988;318: 1423-31.

19. Lowe GD, Upton MN, Rumley A, McConnachie A, O'Reilly DS, Watt GC. Different effects of oral and transdermal hormone replacement therapies on factor IX, APC resistance, t-PA, PAI and $\mathrm{C}$-reactive protein: a cross-sectional population survey. Thromb Haemost 2001;86:550-6.

20. Tan EM, Cohen AS, Fries JF, Masi AT, McShane DJ, Rothfield $\mathrm{NF}$, et al. The 1982 revised criteria for the classification of systemic lupus erythematosus. Arthritis Rheum 1982;25:1271-7.

21. Hochberg MC. Updating the American College of Rheumatology revised criteria for the classification of systemic lupus erythematosus [letter]. Arthritis Rheum 1997;40:1725.

22. Ognenovski VM, Marder W, Somers EC, Johnston CM, Farrehi JG, Selvaggi SM, et al. Increased incidence of cervical intraepithelial neoplasia in women with systemic lupus erythematosus treated with intravenous cyclophosphamide. J Rheumatol 2004;31:1763-7.

23. Ramsey-Goldman R, Mattai SA, Schilling E, Chiu YL, Alo CJ, Howe HL, et al. Increased risk of malignancy in patients with systemic lupus erythematosus. J Investig Med 1998;46:217-22.

24. Bromberger JT, Matthews KA, Kuller LH, Wing RR, Meilahn EN, Plantinga P. Prospective study of the determinants of age at menopause. Am J Epidemiol 1997;145:124-33.

25. Lisabeth LD, Harlow SD, Gillespie B, Lin X, Sowers MF. Staging reproductive aging: a comparison of proposed bleeding criteria for the menopausal transition. Menopause 2004;11:186-97.

26. Waxman JH, Ahmed R, Smith D, Wrigley PF, Gregory W, Shalet $\mathrm{S}$, et al. Failure to preserve fertility in patients with Hodgkin's disease. Cancer Chemother Pharmacol 1987;19:159-62.

27. Contreras G, Pardo V, Leclercq B, Lenz O, Tozman E, O’Nan P, et al. Sequential therapies for proliferative lupus nephritis. N Engl J Med 2004;350:971-80.

28. Boumpas DT, Austin HA III, Vaughn EM, Klippel JH, Steinberg $\mathrm{AD}$, Yarboro $\mathrm{CH}$, et al. Controlled trial of pulse methylprednisolone versus two regimens of pulse cyclophosphamide in severe lupus nephritis. Lancet 1992;340:741-5.

29. Hoffman GS, Leavitt RY, Fleisher TA, Minor JR, Fauci AS. Treatment of Wegener's granulomatosis with intermittent highdose intravenous cyclophosphamide. Am J Med 1990;89:403-10. 
30. Riis BJ, Christiansen C, Johansen JS, Jacobson J. Is it possible to prevent bone loss in young women treated with luteinizing hormone-releasing hormone agonists? J Clin Endocrinol Metab 1990; 70:920-4.

31. Friedman AJ, Hoffman DI, Comite F, Browneller RW, Miller JD, and the Leuprolide Study Group. Treatment of leiomyomata uter with leuprolide acetate depot: a double-blind, placebo-controlled, multicenter study. Obstet Gynecol 1991;77:720-5.

32. Laufer MR, Townsend NL, Parsons KE, Brody KA, Diller LR, Emans SJ, et al. Inducing amenorrhea during bone marrow transplantation: a pilot study of leuprolide acetate. J Reprod Med 1997:42:537-41.

33. Matta WH, Shaw RW, Hesp R, Evans R. Reversible trabecular bone density loss following induced hypo-oestrogenism with the GnRH analogue buserelin in premenopausal women. Clin Endocrinol (Oxf) 1988;29:45-51.

34. Paoletti AM, Serra GG, Cagnacci A, Vacca AM, Guerriero S, Solla E, et al. Spontaneous reversibility of bone loss induced by gonadotropin-releasing hormone analog treatment. Fertil Steril 1996;65:707-10.
35. Dawood MY, Ramos J, Khan-Dawood FS. Depot leuprolide acetate versus danazol for treatment of pelvic endometriosis: changes in vertebral bone mass and serum estradiol and calcitonin. Fertil Steril 1995;63:1177-83.

36. Swaak A, van den Brink R, Smeenk R, Manger K, Kalden J, Tosi $\mathrm{S}$, et al. Systemic lupus erythematosus: clinical features in patients with a disease duration of over 10 years, first evaluation. Rheumatology (Oxford) 1999;38:953-8.

37. Sen D, Keen RW. Osteoporosis in systemic lupus erythematosus: prevention and treatment. Lupus 2001;10:227-32.

38. Practice Committee of the American Society for Reproductive Medicine. Ovarian tissue and oocyte cryopreservation. Fertil Steril 2004;82:993-8.

39. Chapman RM, Sutcliffe SB. Protection of ovarian function by oral contraceptives in women receiving chemotherapy for Hodgkin's disease. Blood 1981:58:849-51.

40. Longhi A, Pignotti E, Versari M, Asta S, Bacci G. Effect of oral contraceptive on ovarian function in young females undergoing neoadjuvant chemotherapy treatment for osteosarcoma. Oncol Rep 2003;10:151-5. 